\title{
TRANSVAGINAL ULTRASONOGRAPHY IS THE DIAGNOSTIC METHODFOR EVALUATION OF ABNORMAL UTERINE BLEEDING
}

Kulsum Haq ${ }^{1}$. Shabnom Ferdous Chowdhury ${ }^{2}$. Maksuda Mannan ${ }^{3}$. Rokshana Ivy ${ }^{4}$, K. Shehnecla Tasnim ${ }^{5}$

\begin{abstract}
:
Objective: The objective of this study is to assess the utility of transvaginal iiltrasonography in patients presenting abnormal uterine bleeding

Materials and methods: Between July 2000 to December 2000, 50 patients underwent transvaginal sonography (TVS) for abnormal uterine bleeding. All patients had transvaginal sonography before histopathology. On TVS, the sonographic appearance of the endometrium was classified according to the following parameters: normal or abnormal thickness, homogeneous or heterogeneous echogenicity, bulbous contour, discontinuous, or obscured. A comparison was performed between TVS with histopathology.
\end{abstract}

Results: The most common causes for the abnormal uterine bleeding were leiomvoma in TVS (42.0\%). Other causes were dysfunctional uterine bleeding (28.0\%), adenomyosis (6.0\%). endometrialpolyp (4.0\%), thin atrophic endometrium (8.0\%), endometrial hyperplasia (8.0\%). and endometrial carcinoma (4.0\%). After histopathologic evaluation $40.0 \%$ patients had leiomvoma, $24.0 \%$ DUB, $10.0 \%$ adenomyosis, $8.0 \%$ endometrial polyp, $8.0 \%$ thin atrophic endometrium, $6.0 \%$ endometrial carcinoma and $4.0 \%$ endometrial hvperplasia. Comparing the TVS with histopathologic findings, TVS had a sensitivity of $94.3 \%$, specificity of $80.0 \%$, accuracy of $90.0 \%$, PPVof91.6\% andNPVof $85.7 \%$.

Conclusion: TVS is an easy, safe, rapid and tolerable procedure. It has an excellent diagnostic accuracy in the diagnosis of uterine pathology responsible for abnormal uterine bleeding.

Abbreviation: TVS transvaginal sonographv; AUB abnormal uterine bleeding; DUB dysfunctional uterine bleeding, $P P V$ positive predictive value, NPV Negative predictive value.

Key words: Transvaginal sonography, endometrium

\section{Introduction:}

Abnormal uterine bleeding is one of the most common gynaecological problems and may be defined as any

1. Dr. Kulsum Haq. Assistant Professor (C.C). Department of Gynaecology and Obstetrics. Shahccd Suhrawardy Medical College. Dhaka

2. Dr. Shabnom Ferdous Chowdhury. Associate Professor (C.C). Department of Gynaecology and Obstetrics, Shaheed Suhrawardy Medical College. Dhaka

3. Dr. Maksuda Mannan. Specialist, Department of Radiology and Imaging. United Hospital. Dhaka

4. Dr. Rokshana Ivy, Senior Consultant (Gynaecology and Obstetrics) Maternal and Child Health Training Institute, Azimpur. Dhaka.

5. Dr. K. Shehneela Tasnim. Medical officer (Gynaecology and Obstetrics). Shahid

Suhrawardy Hospital. Dhaka

Correspondence:

Dr. Kulsum Haq

Assistant Professor (C.C). Department of Gynaecology and Obstetrics. Shaheed

Suhrawardy Medical College. Dhaka bleeding from uterus that differ from that of usual normal menstrual bleeding in frequency of occurrence or in amount or in alteration of flow ${ }^{1}$. It can occur in pre-. periand post-menopausal women and even in some women in reproductive age. Number of women seeking medical attention for abnormal and excessive uterine bleeding (AUB) has been increasing ${ }^{2}$. Determination of cause of AUB is essential in planning of appropriate therapy. The diagnostic aid for assessing AUB are cervical biopsy. endometrial curettage, laparoscopy, hysteroscopy and ultrasonogram, including transvaginal ultrasonogram. New vaginal probe ultrasonographic transducers are of higher frequency and closer proximity to structures being studied. This result is excellent in near-field resolution $\mathrm{w}$ ith high degree of magnification, thus yielding a type of sonomicroscopy. Previously investigators used highresolution abdominal ultrasonographic equipment to assess uterine content. It is well known that the phasic variation in endometrial thickness can be easily visualized by transvaginal ultrasonography. Intrauterine spaceoccupying lesions including submucous myomas, endometrial polyp and cancer can also be accurately diagnosed by transvaginal ultrasonography and their location and size also delineated ${ }^{3}$. TVS examination of 
uterus in AUB women is very quick, simple, painless, less expensive and accurate.

In view of the above considerations, it is important to attempt to rationalize the diagnostic approach in this group of women. Thus we performed a prospective six months study on a series of patients referred for abnormal uterine bleeding. The aims of the study were to verify the reliability of transvaginal ultrasonography in diagnosis of uterine pathology in patients with abnormal uterine bleeding (AUB) and to determine the feasibility, acceptability and validity of TVS in patients with AUB.

\section{Materials and Methods:}

In the period July 2000 to December 2000 all women referred for abnormal uterine bleeding to the Department of Obstetrics and Gynaecology, Dhaka Medical College Hospital (DMCH), Dhaka were considered for inclusion in the study. In this series fifty cases were enrolled randomly. Most of the patients had already undergone unsuccessful medical treatments. Excessive uterine bleeding was diagnosed based on the history, serum haemoglobin and iron concentrations.

Patients with an intrauterine device, who had received hormonal treatment in the previous 3 months or who had already undergone dilatation and curettage or diagnostic or operative hysteroscopy, who had obstetric cause of vaginal bleeding, known cases of carcinoma cervix, who had bleeding disorder were excluded from the analysis.

Transvaginal sonography (TVS) was conducted in the Department of Radiology and Imaging, Bangladesh Institute of Research and Rehabilitation in Diabetes, Endocrine and Metabolic Disorders (BIRDEM), Dhaka by a specialist Radiologist.

The endometrial cavity contour and myometrium were studied by US probe from internal os to the fundus in longitudinal and transverse planes. A normal endometrium and uterine cavity were defined by an echo-dense line in the middle of the uterus with a homogenous endometrial lining with distinct margins to the myometrium. Normal myometrium was also uniform in echo pattern. Any discontinuity was noted and all other findings, such as deformations of endometrial lining, absence of the central echo-dense line, any mass lesion with or without well-defined margins or variable echo-density were considered to be abnormal. The maximum endometrial thickness was measured in longitudinal plane. In normal sonograms, the endometrial linings were apposed, by definition, and the total double-layer thickness was measured. The findings of transvaginal ultrasonography were confirmed by histological examinations of the specimen obtained by resection after hysterectomy or curettage. The biopsy specimens were collected and preserved in $10 \%$ formalin and sent for histopathology. Gross examinations of excised uterus were done. Two to four biopsies were sent in capsule and transferred on tissue autoprocessor for 14-16 hours and processed for routine paraffin sectioning stained with haematoxylin. eosin. alcohol, distilled water and xylin. On microscopic examinations, any alteration in gland architecture, epithelial growth pattern and smooth muscle cytology were recorded.

The sensitivity, specificity and positive and negative predictive values (PPV. NPV) of transvaginal ultrasonography in detecting the presence of a uterine pathology were calculated considering the histopathological finding as the true diagnosis.

Evaluation of predictive power was based on sensitivity= true positive (TP) X 100/TP + false negatives (FN), specificity $=$ true negatives $(\mathrm{TN}) \mathrm{X} 100 / \mathrm{TN}+$ false positives (FP), positive predictive value $(\mathrm{PPV})=$ TPX100/TP+FP. negative predictive value $(\mathrm{NPV})=\mathrm{TN}$ $\mathrm{X} 100 / \mathrm{TN}+\mathrm{FN}$. diagnostic accuracy $(\mathrm{DA})=\mathrm{TP}+\mathrm{TN} \mathrm{X}$ 100 total.

\section{Results:}

In the 6 months period considered, 50 women underwent clinical, ultrasound and histopathological evaluation for abnormal uterine bleeding. Mean age of the patients was 37.4 years. The highest incidence of AUB was prevalent in cases below 40 years. This series included 40 patients who had premenopausal and 10 had post menopausal bleeding. The most common causes for the abnormal uterine bleeding were leiomyoma in transvaginal ultrasonography (42.0\%). Other causes were dysfunctional uterine bleeding (28.0\%). adenomyosis (6.0\%), endometrial polyp (4.0\%). thin atrophic endometrium (8.0\%), endometrial hyperplasia (8.0\%), and endometrial carcinoma (4.0\%). After histopathologic evaluation $40.0 \%$ patients had leiomyoma, $24.0 \%$ DUB, $10.0 \%$ adenomyosis, $8.0 \%$ endometrial polyp. $8.0 \%$ thin atrophic endometrium, $6.0 \%$ endometrial carcinoma and $4.0 \%$ endometrial hyperplasia. Among all patients, 33 were true positive, 3 false positive. 12 true negative and 2 false negative (Table I). Comparing the TVS with histopathologic findings, TVS had a sensitivity of $94.3 \%$, specificity of $80.0 \%$, accuracy of $90.0 \%$, PPV of $91.6 \%$ and NPV of $85.7 \%$ (Table: II)

\section{Table-I:}

Comparison of TVS with histopathologic findings in a series of 50 consecutive women with abnormal uterine bleeding 


\begin{tabular}{|l|l|l|l}
\hline \multirow{2}{*}{ TVS finding } & \multicolumn{2}{|l|}{ Histopathologic finding } & \multirow{2}{*}{ Total } \\
\cline { 2 - 4 } & Abnormal & Normal & \\
\hline Abnormal & 33 & 3 & 36 \\
\hline Normal & 7 & 12 & 14 \\
\hline Total & 35 & 15 & 50 \\
\hline
\end{tabular}

In our study, use of transvaginal ultrasonography in delineating the cause of abnormal uterine bleeding had $94.3 \%$ sensitivity, $80 \%$ specificity, $90 \%$ accuracy, $91.6 \%$ positive predictive value and $85.7 \%$ negative predictive value. Other investigators, such as Dijkhuizen et al. ${ }^{7}$ had indicated similar diagnostic accuracy (96\% sensitivity and $89 \%$ specificity) when routine vaginal probe ultrasonography and histopathology were compared in the evaluation of women with abnormal uterine bleeding. In Williams and Marshburn ${ }^{6}$ series vaginal probe ultrasonography detected intrauterine lesions with a sensitivity of $67 \%$, specificity $93 \%$, positive predictive value $80 \%$ and negative predictive value $86 \%$.

\begin{tabular}{|l|l|}
\hline Predictive characteristics of & Percentage \\
\hline Sensitivity & 94.3 \\
\hline Specificity & 80.0 \\
\hline Accuracv & 90.0 \\
\hline PPV & 91.6 \\
\hline NPV & 85.7 \\
\hline
\end{tabular}

\section{Discussion:}

Abnormal vaginal bleeding is a common presenting complaint in both pre-, peri- and postmenopausal women, and its management differs according to the presence or absence of identifiable uterine abnormalities. Although numerous articles in the literature point to using conventional transvaginal pelvic sonography as a screening procedure for patients who require further diagnostic evaluation ${ }^{4,5}$, our study suggests that a small number of patients with treatable abnormalities may be inadvertently directed away from further evaluations on the basis of normalappearing endometrium on conventional transvaginal sonography.

TVS is very sensitive for detecting leiomyoma, dysfunctional uterine bleeding, adenomyosis and endometrial carcinoma. But TVS alone is not sufficient in the evaluation of endometrial polyps as a cause of abnormal uterine bleeding as it can be confused with endometrial hyperplasia. Two cases of endometrial polyp were missed in this study. Hydrosonography was not done in this work for better evaluation of intracavitary lesion, such as endometrial polyps. But TVS with simultaneous hydrosonography is most suitable method of diagnosis in such cases as described in one study ${ }^{6}$, but it is not practiced in Bangladesh.

\section{Conclusion:}

TVS is an advanced enhancement of conventional US. It is an easy, safe, rapid and tolerable procedure. It has an excellent diagnostic accuracy in the diagnosis of uterine pathology responsible for abnormal uterine bleeding. It can evaluate the endoluminal abnormalities more definitely. Hence, it may then direct biopsy procedures and minimize false negative results, but it cannot substitute tissue diagnosis.

\section{Reference:}

1. Indman PD. Abnormal uterine bleeding: accuracy of vaginal probe ultrasound in predicting abnormal hysteroscopic findings. J Rcprod Med 1995:40:545-8.

2. Dubinsky TJ, Parvey HR. Maklad N. The role of transvaginal sonography and endometrial biopsy in the evaluation of peri- and postmenopausal bleeding. Am J Radiol 1997: 169:145-9.

3. Long CA. Evaluation of patients with abnormal uterine bleeding. Am J Obstet Gynecol 1996: 175:784-6.

4. Emmanuel M. Verdel M. Wamsteker K.. Lammes FB. A prospective comparison of transvaginal ultrasonography and diagnostic cases a biopsy sample is always obtained. Furthermore, hysterohysteroscopy in the evaluation of patients with abnormal uterine bleeding: scopy did not always correctly differentiate myomas from clinical implications. Am. J. Obstet. Gynecol. 1995:172:547-552.

5. Goldstein SR. Zeltser I. Horan CK. Snyder JR. Schwartz LB. Ultrasonography-based triage for perimenopausal patients with abnormal uterine bleeding. Am J Obstet Gynecol 1997:177:102-108

6. Williams CD. Marshburn PB. A prospective study of transvaginal hydrosonography in the evaluation of abnormal uterine bleeding. Am J Obstet Gynecol 1998: 179:292-8.

7. Dijkhuizen FP. Brolmann HA. Potters AE. Bongers MY. Hientz PM. The accuracy of transvaginal ultrasonography in the diagnosis of endometrial abnormalities. Obstet Gvnecol 1996: 87:345-9 